Many hospitals don't offer NTC, which is therefore done in the community - however certain underlying health conditions mean NTC with access to specialist services occurs.

Whilst neither condoning, nor criticising this, we wanted to ascertain NTC safety in 'high-risk' children, and to assess the quality of consent given by both parents as the BMA and BAPS suggest.

Methods Retrospective 2-year case-note review (2009-10) of circumcisions in a tertiary children's hospital. Hospital admissions/ surgical databases searched and NTC medical notes interrogated for consent forms, notes entries and other indicators of explanations to parents/family together with medical and surgical complications and outcomes. Whilst demographic information, ethnic origin, religion and underlying medical condition recorded, only the last is reported here.

Results 199 circumcisions (all male) were performed. Exclusions: 181 part of larger procedure/medically indicated $=18$ NTC. Age $4.5+/-4.0$. Underlying conditions: Bleeding disorder 6, cardiac defect 5 , respiratory problem 3 , renal failure 1 , leukaemia 1 , vitamin B12 deficiency 1 , megalocornea 1

Consent record: Risk documented: Generic bleeding (100\%), infection ( $96 \%$ ), $<5 \%$ all others (serious haemorrhage, sepsis, fistula, penile loss, GA risks). No mention of risks re underlying disease. No record of both parents consenting.

Outcomes of procedure: Readmissions 0, Length of stay: mean 1.8 days (1-4), $60 \%$ day case. 4 stayed 3-4 days (3 bleeding disorders 2 transexamic acid cover)

Complications 1) procedure: None 16, facial scratch 1, tooth lost 1 ; 2) post procedure: None 11 , ooze/mild wound bleeding 3 , moderate bleeding 1, bradycardic during recovery 2 , mild swelling/ moderate pain 1 , fever/cough 1 . All resolved without long-term effects.

Conclusion Whilst NTC in 'high-risk' children is relatively safe in our specialist centre, with only minor self-limiting complications, consent documentation is poor. Although verbal explanation might have been better than that recorded inadequate recording of those risks discussed and the absence of documented consent from both parents is medico-legally problematic.

Standardization of NTC risks explained to families and consent records from both parents must be ensured.

\section{G107 ETHICAL DILEMMAS IN RESUSCITATION OF BORDERLINE VIABILITY INFANTS: DECISION-SUPPORT FOR CLINICIANS}

doi:10.1136/archdischild-2013-304107.119

EG Brown. King's College London, London, UK

Infants born at the borderline of viability pose difficult ethical questions because of poor survival rates and the possibility of long-term disability ${ }^{1}$.

The Nuffield Council guidelines for resuscitation practice ${ }^{2}$ emphasise the need to consider each case individually.

The four principles approach to medical ethics ${ }^{3}$ provides a basis for individual case decision-making as follows:

The principle of respect for autonomy recognises the rights and responsibilities of parents as surrogate decision-makers, although this right is qualified by the need to act in the best interests of their child;

The principles of beneficence and non-maleficence prioritise the 'best interests' standard by incorporating quality-of-life assessments, and if necessary by limiting the authority of the parents;

The principle of justice addresses the wider result of decisions and the interests of the child within the family.

The role of the clinician within the clinician/parent partnership is twofold: firstly to inform the parents by communicating clinical knowledge and experience, and secondly to provide support for the family through professional values and trust.

Conclusion Weighting the four principles of medical ethics with a focus on the best interests of the child provides a flexible and simple means to address each borderline viability infant case on an individual basis and reach the best ethical solution.

\section{REFERENCES}

1. Moore T, Hennessey EM, Myles J, Johnson SJ, Draper ES, Costeloe KL, Marlow N. Neurological and developmental outcome in extremely preterm children born in England in 1995 and 2006: the EPICure studies. BMJ 2012; 345: e7961

2. Nuffield Council on Bioethics. Dilemmas in current practise: babies born at the borderline of viability. Critical care decisions in foetal and neonatal medicine: ethical issues. London: Nuffield Council; 2006. http://www.nuffieldbioethics.org/neonatal- medicine (accessed 27/09/2012) p 67-87

3. Gillon R. Medical ethics: four principles plus attention to scope. BMJ 1994; 309 184-188.

\section{G108 IMPACT OF SLEEP SYSTEMS (SS) ON POSTURE AND OUALITY OF LIFE (OOL) IN CHILDREN WITH NEUROLOGICAL DISABILITIES}

doi:10.1136/archdischild-2013-304107.120

${ }^{1} \mathrm{H}$ Royden, ${ }^{1} \mathrm{R}$ Mithyantha, ${ }^{2} \mathrm{~S}$ Clarke, ${ }^{2} \mathrm{~J}$ Birch, ' $\mathrm{Z}$ Bassi. 'Neurodisability Department, Community Child Health, Alder Hey Children's NHS Foundation Trust, Liverpool, UK ${ }^{2}$ Community Physiotherapy Department, Alder Hey Children's NHS Foundation Trust, Liverpool, UK

Aims Children with neurodisability and limited mobility are at an increased risk of postural deformity which impacts on their overall health and QOL. SS are used as part of an individualised posture management programme for children with restricted mobility. There is limited research evidence on the impact of SS on hip stability, body symmetry and QOL of the child and family. There are also financial implications associated with use of SS. We aim to assess the impact of SS in children seen in a single tertiary centre.

Methods Clinical data was retrospectively collated from all the children who had SS introduced between April 2010 to April 2011. Data collection included measurements of hip radiographs (HR), Goldsmiths indices for body symmetry (GI), hip abduction measurements (HAM) and QOL measure (CPChild questionnaire) at baseline and twelve months post-intervention. These evaluations are part of our routine care plans and trust hip surveillance protocol.

Results Of the 61 children offered SS, 58 agreed to use them. [M; $\mathrm{F}=30: 28$; Median(range) age $-9.17(0.5$ to 19.25$)$ years]. The diagnosis was CP in $43(74.1 \%)$ children while $15 / 58(25.9 \%)$ were identified with non-CP causes including genetic (Retts, Lissencephaly,) and neurodegenerative condition (PEHO).

The clinical parameters improved or remained stable in the majority of children at twelve months (HR-92\%; GI-65\%, HAM-66\%). In subgroup analysis the improvement/stability was analysed in CP vs. non-CP group in each of the clinical categories - HR [20/21(95.2\%) v $5 / 6(83.3 \%), p=0.33]$; GI $[18 / 22(81.8 \%)$ v $3 / 10(30 \%), p=0.004]$, HAM [14/22(63.6\%) v 4/5 (80\%),p=0.48]. Ten parents/carers completed serial QOL questionnaires. Overall scores were stable/ improved in $66.7 \%$ children. Subcategory data showed improvement or stability in $5 / 5(100 \%)$, personal care/ADL, $3 / 5(60 \%)$ in positioning/transferring, $2 / 2(100 \%)$ in comfort/emotions, $7 / 10(70 \%)$ in health scores. No differences recorded between the two subgroups.

Conclusion In our patient group, we observed a trend towards improvement in clinical parameters and QOL following introduction of SS. The improvement was most significant in body symmetry (GI) in children with CP. Further research is required to assess long term benefits of SS. 